\title{
Introduction to the Minitrack Behavioral Economics in the Digital Economy: Digital Nudging and Interface Design
}

\author{
Markus Weinmann \\ Erasmus University Rotterdam \\ weinmann@rsm.nl
}

\author{
Christoph Schneider \\ IESE Business School \\ cschneider@iese.edu
}

Research in psychology and behavioral economics has repeatedly demonstrated that people act in boundedly rational ways, and their decision making is influenced by various heuristics and biases, either for the good or bad: Heuristics can aid decision making when people face simple, recurrent problems; they simplify problem-solving by reducing the amount of information to be processed. However, heuristics can also lead to cognitive biases and introduce systematic errors when people face complex decisions.

Examining the effects of psychological, cognitive, emotional, and social factors on judgment and decision making, research has repeatedly shown that the context matters when making judgments or decisions. People's decisions are not only influenced by the content of the choices, but also the way the choices are presented, and "nudges ${ }^{1}$," such as setting defaults or framing decisions, can influence people's behavior substantially.

Today, choices are increasingly made in digital contexts, thus it is important to understand the psychological effects of user-interface design on people's choices. In particular, it is important to understand how "digital nudging" influences online decision making.

The main purpose of this minitrack is to explore and extend, as well as exchange, innovative research related to online decision making in the context of information systems design. In particular, this minitrack aims to examine the main applications of behavioral interventions and digital nudges in information-systems design, in particular, research with an emphasis on the effects of interface design on users' behavior, judgment, and decision making in online environments.

This year marks the fourth edition of the minitrack on behavioral economics and digital nudging. While findings from behavioral economics have received much attention, digital applications of behavioral economics—e.g., digital nudging — are just beginning to

\footnotetext{
${ }^{1}$ Thaler, R.H., \& Sunstein, C.R. (2008). Nudge: Improving Decisions about Health, Wealth, and Happiness. Yale University Press, New Haven \& London.

${ }^{2}$ Weinmann, M., Schneider, C., \& vom Brocke, J. (2016). "Digital Nudging," Business \& Information Systems Engineering, 58(6): 433-436.
}

be explored. As information systems allow to dynamically adapt and personalize content, they provide unique opportunities to implement concepts from behavioral economics. Our goal is to provide a platform to discuss cutting-edge research on digital nudging and online decision making.

The three papers accepted for this year's minitrack span a range of topics related to digital nudging. In the first paper, titled "Sensitive to the Digital Touch? Exploring Sensory Processing Sensitivity and Its Impact on Anthropomorphized Products in E-Commerce," Lingyao Yuan and Jordan Barlow examine how individuals receive, process, and react to sensory information in online bidding. The authors find that people's sensory processing sensitivity positively influences the willingness to pay. In the second paper, titled "Digital Nudging for Technical Debt Management: Insights from a Technology-driven Organization," Lorena Buchmann and Kazem Haki examine how software developers can be prevented to sacrifice software quality for short-term goals, also known as technical debt. The authors propose nudging design principles for technical debt management to encourage software developers to focus on long-term goals. In the final paper, titled "The Dark Side of Privacy Nudging-An Experimental Study in the Context of a Digital Work Environment," Torben Barev, Melanie Schwede, and Andreas Janson tested two digital privacy nudges - social and framing nudge - to discourage users to disclose less personal information. Surprisingly, the results indicate that privacy nudges negatively influence information disclosure behavior, and that users perceive them as a threat.

We would like to thank the researchers who submitted their work to this minitrack. We also thank the many reviewers for their outstanding contributions and their help in ensuring the quality of the papers in this minitrack.

Schneider, C., Weinmann, M., \& vom Brocke, J. (2018). "Digital Nudging-Influencing Choices by Using Interface Design," Communications of the ACM, 61(7), 67-73. 